\title{
Evidence for cosmic neutrino background from CMB circular polarization
}

\author{
Rohollah Mohammadi ${ }^{\mathrm{a}}$ \\ School of Physics, Institute for Research in Fundamental Sciences (IPM), Tehran, Iran
}

Received: 11 December 2013 / Accepted: 19 September 2014 / Published online: 17 October 2014

(C) The Author(s) 2014. This article is published with open access at Springerlink.com

\begin{abstract}
The primordial anisotropies of the cosmic microwave background are linearly polarized via Comptonscattering. On the other hand, a primordial degree of circular polarization of the Cosmic Microwave Background is not observationally excluded. In this work, we discuss the generation of the circular polarization of $\mathrm{CMB}$ via their scattering on the cosmic neutrino background since the epoch of recombination. We show that the photon-neutrino interaction can transform plane polarization into circular polarization through processes $\gamma+v \rightarrow \gamma+v$ and the Stokes- $V$ parameter of $\mathrm{CMB}$ has a linear dependence on the wavelength and square dependence on the average bulk velocity of the cosmic neutrino background and also the maximum value of $C^{V}$ is estimated in range of a few $\mathrm{nK}$ square.
\end{abstract}

\section{Introduction}

Modern cosmological observations of the Cosmic Microwave Background (CMB) radiation contain valuable information about our universe. The CMB photons have decoupled from matter about $3 \times 10^{5}$ years after the Big-Bang (BB), so we are unable to probe the universe closer than 300,000 years to the $\mathrm{BB}$ by using $\mathrm{CMB}$. Cosmological information encoded in the $\mathrm{CMB}$ radiation concerns not only temperature fluctuations and the spectrum of anisotropy pattern, but also the intensity and spectrum of linear and circular polarizations. From a result of the anisotropic Compton scattering around the epoch of recombination, it is generally expected that some relevant linear polarizations (about $10 \%$ ) of CMB radiation should be present [1-4], and polarization fluctuations are smaller than the temperature fluctuations [5]. Currently, there are several ongoing experiments [6-12] attempting to measure CMB polarizations. Theoretical studies of CMB polarizations were carried out in Refs. [1-4,13], and numerical calculations [14-16] have confirmed that about $10 \%$ of the

\footnotetext{
a e-mail: rmohammadi@ipm.ir
}

CMB radiation is linearly polarized, via the Compton and Thompson scattering of unpolarized photons at the last scattering surface (the redshift $z \sim 10^{3}$ ). Polarized light is conventionally described in terms of the Stokes parameters, a linearly polarized radiation is described by nonzero values for the Stokes parameters $Q$ and/or $U$ and the possibility of the generation of circular polarization can be determined by the Stokes parameter $V$ [17]. On the basis of the mechanism discussed in [1], the linear polarization of the CMB in the presence of a large-scale magnetic field $B$ can be converted to the circular polarization under the formalism of the generalized Faraday rotation (FR) [18,19] known as the Faraday conversion (FC). The evolution of the Stokes parameter $V$ given by this mechanism is obtained as

$\dot{V}=2 U \frac{d}{d t}\left(\Delta \phi_{F C}\right)$

where $\Delta \phi_{F C} \propto B^{2}$ is the Faraday conversion phase shift [20]. There are several papers which have attempted to discuss the probability of the generation of circular polarization of CMB photons. Giovannini has shown that if the CMB photons are scattered via electrons in the presence of a magnetic field, a non-vanishing $V$ mode can be produced [21,22]. Furthermore, Cooray, Melchiorri and Silk have discussed that the $\mathrm{CMB}$ radiation observed today is not exactly the same as the field last scattered [20], Bavarsad et al. have shown that CMB polarization acquires a small degree of circular polarization when a background magnetic field is considered or the quantum electrodynamic sector of the standard model is extended by Lorentz non-invariant operators as well as non-commutativity [23], Motie and Xue have discussed that the circular polarizations of radiation fields can be generated from the effective Euler-Heisenberg Lagrangian [24] and the transform plane polarization into circular polarization via photon-photon interactions mediated by the neutral hydrogen background, $\gamma+\gamma+$ atom $\rightarrow \gamma+\gamma+$ atom, through completely forward processes, has been discussed by Sawyer [25]. We would like to point out that photon- 
neutrino scattering can generate circular polarization. The reason for that is: in context of the standard model we have the purely left-handed interaction for neutrinos which caused linearly polarized photons achieve circular polarizations by interacting with left-handed neutrinos, in contrast they do not acquire circular polarizations by interacting with electrons in the forward scattering terms of [1]. We can consider any linear polarization as two equal component, left- and righthanded circular polarization. Due to the left-handed interaction of the neutrino, only one part of this linear polarization (left-handed component) is affected by neutrino. Finally after neutrino-photon scattering, the total number of left- and right-handed circular polarization become different and we have a net circular polarization for photons. In this study, we are going to check and calculate the generation of circular polarization for $\mathrm{CMB}$ due to their scattering with the cosmic neutrinos background $(\mathrm{C} v \mathrm{~B})$.

On the other hand, a similar probe like the $\mathrm{CMB}$ is the cosmic neutrino background $(\mathrm{C} v \mathrm{~B})$ which can give us very helpful information about the early universe. Due to their weak interaction they decouple earlier, about 1 second after the $\mathrm{BB}$ from matter at a temperature of $T_{v} \approx 1 \mathrm{MeV}\left(10^{10}\right.$ $\mathrm{K})$. $\mathrm{C} v \mathrm{~B}$ of today (with temperature $T_{0 v} \approx 1.95 \mathrm{~K}$ ) therefore contains information of the universe already 1 second after the $\mathrm{BB}$. But we should remind the reader that the detection of this $\mathrm{C} v \mathrm{~B}$ seems to be hardly possible due to the weak interaction of the neutrinos with matter and due to their low energy. Nevertheless, several methods have been discussed in the literature to search for these relic neutrinos [26-36]. Here we discuss the possibility to find any effects of $C \nu B$ on the circular polarization of the CMB photons via photonneutrino scattering. As is well known, the photon-neutrino cross section in the context of the standard model is very small because neutrinos are neutral particles with very small electromagnetic dipole moment $\mu_{v} \propto m_{v}$ and also the leading order of photon-neutrino interaction (one-loop) contain the weak interaction. But this is not really bad news for our idea because we are going to consider the last scattering surface for photon-neutrinos around the epoch of recombination. This means if coherent photon-neutrino forward scattering after recombination age can provide any sources for the circular polarization of CMB, $\Delta \phi_{F C}$ grows due to the large distance (larger than $\mathrm{Mpc}$ ) or equivalently the large time scale of evolution (see Eq. 1). In principle, by the effects of background fields, particle scattering, and temperature fluctuations, linear polarizations of the CMB radiation field propagating from the last scattering surface can rotate each other and convert to circular polarizations. In this study we will study the distribution of the neutrino-photon scattering as regards the generation of the circular polarization of the CMB. First we give a brief introduction to the Stokes parameters and derive the time evolution of these parameters in terms of the photon-particle scattering. Then by considering the weak and electrodynamic interactions, we will find the time evolution of the Stokes parameters in terms of photonneutrino interactions. Finally we try to estimate the maximum value of the $V$-mode polarization by using the relevant values of energy and number density of the cosmic neutrinos around the recombination epoch.

\section{Stokes parameters}

As usual, we characterized the polarization of CMB by means of the Stokes parameters of radiation: $I, Q, U$ and $V$. Assume a quasi-monochromatic electromagnetic wave propagating in the $\hat{z}$-direction which is described by

$E_{x}=a_{x}(t) \cos \left[\omega_{0} t-\theta_{x}(t)\right], \quad E_{y}=a_{y}(t) \cos \left[\omega_{0} t-\theta_{y}(t)\right]$,

where amplitudes $a_{x, y}$ and phase angles $\theta_{x, y}$ are slowly varying functions with respect to the period $\mathcal{T}_{0}=2 \pi / \omega_{0}$. Stokes parameters, which describe polarization states of a nearly monochromatic electromagnetic wave, are defined as the following time averages [17]:

$$
\begin{aligned}
& I=\left\langle a_{x}^{2}\right\rangle+\left\langle a_{y}^{2}\right\rangle, \\
& Q=\left\langle a_{x}^{2}\right\rangle-\left\langle a_{y}^{2}\right\rangle, \\
& U=\left\langle 2 a_{x} a_{y} \cos \left(\theta_{x}-\theta_{y}\right)\right\rangle, \\
& V=\left\langle 2 a_{x} a_{y} \sin \left(\theta_{x}-\theta_{y}\right)\right\rangle,
\end{aligned}
$$

where the parameter $I$ is total intensity, $Q$ and $U$ intensities of linear polarizations of electromagnetic waves, whereas the $V$ parameter indicates the difference between left- and right- circular polarizations intensities. Linear polarization can also be characterized through a vector of modulus $P_{L} \equiv$ $\sqrt{Q^{2}+U^{2}}$. The time evolution of these Stokes parameters is given through the Boltzmann equation. The Boltzmann equation is a systematic mechanism in order to describe the evolution of the distribution function under gravity and collisions. One can consider each polarization state of the CMB radiation as a phase space distribution function $\xi$. The classical Boltzmann equation generally is written as

$\frac{d}{d t} \xi=\mathcal{C}(\xi)$

where the left-hand side is known as the Liouville term; it deals with the effects of gravitational perturbations about the homogeneous cosmology. The right-hand side of the Boltzmann equation contains all possible collision terms. By considering the contribution of the neutrino-photon scattering on the right-hand side of the above equation, we calculate the time evolution of the each polarization state of the CMB photons. For the rest of calculations, Stokes parameters are given in a quantum-mechanical description. An arbitrary polarized state of a photon $\left(\left|k^{0}\right|^{2}=|\mathbf{k}|^{2}\right)$, propagating in the $\hat{z}$-direction, is given by 
$|\epsilon\rangle=a_{1} \exp \left(i \theta_{1}\right)\left|\epsilon_{1}\right\rangle+a_{2} \exp \left(i \theta_{2}\right)\left|\epsilon_{2}\right\rangle$,

where the linear bases $\left|\epsilon_{1}\right\rangle$ and $\left|\epsilon_{2}\right\rangle$ indicate the polarization states in the $x$ - and $y$-directions. Quantum-mechanical operators in these linear bases, corresponding to the Stokes parameter, are given by

$\hat{I}=\left|\epsilon_{1}\right\rangle\left\langle\epsilon_{1}|+| \epsilon_{2}\right\rangle\left\langle\epsilon_{2}\right|$,

$\hat{Q}=\left|\epsilon_{1}\right\rangle\left\langle\epsilon_{1}|-| \epsilon_{2}\right\rangle\left\langle\epsilon_{2}\right|$,

$\hat{U}=\left|\epsilon_{1}\right\rangle\left\langle\epsilon_{2}|+| \epsilon_{2}\right\rangle\left\langle\epsilon_{1}\right|$,

$\hat{V}=i\left|\epsilon_{2}\right\rangle\left\langle\epsilon_{1}|-i| \epsilon_{1}\right\rangle\left\langle\epsilon_{2}\right|$.

An ensemble of photons in a general mixed state is described by a normalized density matrix $\rho_{i j} \equiv\left(\left|\epsilon_{i}\right\rangle\left\langle\epsilon_{j}\right| / \operatorname{tr} \rho\right)$, and the dimensionless expectation values for the Stokes parameters are given by

$I \equiv\langle\hat{I}\rangle=\operatorname{tr} \rho \hat{I}=1$,

$Q \equiv\langle\hat{Q}\rangle=\operatorname{tr} \rho \hat{Q}=\rho_{11}-\rho_{22}$,

$U \equiv\langle\hat{U}\rangle=\operatorname{tr} \rho \hat{U}=\rho_{12}+\rho_{21}$,

$V \equiv\langle\hat{V}\rangle=\operatorname{tr} \rho \hat{V}=i \rho_{21}-i \rho_{21}$,

where "tr" indicates the trace in the space of polarization states. These above equations determine the relationship between four Stokes parameters and the $2 \times 2$ density matrix $\rho$ of photon polarization states. In this section, we use notations which used in [24].

\section{The generation of polarized CMB via photon-neutrinos scattering}

The density operators describing a system of photons is given by

$\hat{\rho}=\frac{1}{\operatorname{tr}}(\hat{\rho}) \int \frac{d^{3} k}{(2 \pi)^{3}} \rho_{i j}(k) a_{i}^{\dagger}(k) a_{j}(k)$,

where $\rho_{i j}(k)$ is the general density matrix (7)-(10) in the space of polarization states with a fixed energy-momentum " $k$ ". The number operator $D_{i j}^{0}(k) \equiv a_{i}^{\dagger}(k) a_{j}(k)$. Then the expectation value of this number operator is defined by

$\left\langle D_{i j}^{0}(k)\right\rangle \equiv \operatorname{tr}\left[\hat{\rho} D_{i j}^{0}(k)\right]=(2 \pi)^{3} \delta^{3}(0)\left(2 k^{0}\right) \rho_{i j}(k)$.

On the other hand, the time evolution of the operator $D_{i j}^{0}(k)$, considered in the Heisenberg picture, is

$\frac{d}{d t} D_{i j}^{0}(k)=i\left[H, D_{i j}^{0}(k)\right]$,

where $H$ is the full Hamiltonian. Taking the expectation value of both sides of the above equation gives the Boltzmann equation (4) for the system's density matrix (as well as polarization states) which is a generalization of the usual classical Boltzmann equation for particle occupation numbers. By substituting Eq. (12) in Eq. (13), the time evolution of $\rho_{i j}(k)$ as well as the Stokes parameters is given [1],

$$
\begin{aligned}
& (2 \pi)^{3} \delta^{3}(0)\left(2 k^{0}\right) \frac{d}{d t} \rho_{i j}(k)=i\left\langle\left[H_{I}^{0}(t) ; D_{i j}^{0}(k)\right]\right\rangle \\
& -\frac{1}{2} \int d t\left\langle\left[H_{I}^{0}(t) ;\left[H_{I}^{0}(0) ; D_{i j}^{0}(k)\right]\right]\right\rangle,
\end{aligned}
$$

where $H_{I}^{0}(t)$ is the first order of the interacting Hamiltonian. The first term on the right-handed side is a forward scattering term, and the second one is a higher order collision term. In order to find effects of photon-neutrino scattering on the polarization of the $\mathrm{CMB}$, we start with the following Lagrangian:

$\mathfrak{f}_{I}=\mathfrak{Q E D}_{\mathrm{QED}}+\mathfrak{£}_{e v}$,

where the first term $£_{\mathrm{QED}}$ is the quantum electrodynamic Lagrangian (QED), and the second term $£_{e v}$ is the Lagrangian of the weak interaction containing an electron-neutrino vertex. In the context of the standard model, there is no direct vertex for photon-neutrino scattering; however, the first order of the interaction between photon-neutrino appears during a one-loop interaction where photons and neutrinos both interact with electrons and weak gauge bosons (see Fig. 1).

We express the electromagnetic free gauge field $A_{\mu}$ in terms of plane wave solutions in the Coulomb gauge [37],

$A_{\mu}(x)=\int \frac{d^{3} k}{(2 \pi)^{3} 2 k^{0}}\left[a_{i}(k) \epsilon_{i \mu}(k) e^{-i k \cdot x}+a_{i}^{\dagger}(k) \epsilon_{i \mu}^{*}(k) e^{i k \cdot x}\right]$,

where $\epsilon_{i \mu}(k)$ are the polarization four-vectors and the index $i=1,2$, representing two transverse polarizations of a free photon with four-momentum $k$ and $k^{0}=|\mathbf{k}| \cdot a_{i}(k)\left[a_{i}^{\dagger}(k)\right]$ are the creation [annihilation] operators, which satisfy the canonical commutation relation as follows:

$\left[a_{i}(k), a_{j}^{\dagger}\left(k^{\prime}\right)\right]=(2 \pi)^{3} 2 k^{0} \delta_{i j} \delta^{(3)}\left(\mathbf{k}-\mathbf{k}^{\prime}\right)$.

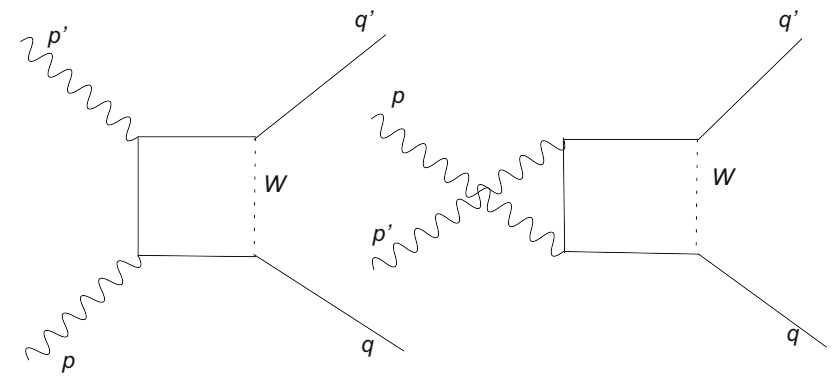

Fig. 1 The typical diagrams of photon-neutrino scattering 
Also the free fermion field $\psi$ is given:

$$
\begin{aligned}
\psi(x)= & \int \frac{d^{3} p}{(2 \pi)^{3}} \frac{1}{\sqrt{2 E_{\mathbf{p}}}} \\
& \times \sum_{r}\left[b_{r}(p) U_{r}(p) e^{-i p \cdot x}+d_{r}^{\dagger}(p) \mathcal{V}_{r}(p) e^{i p \cdot x}\right],
\end{aligned}
$$

where $U_{r}$ and $\mathcal{V}_{r}$ are Dirac spinors, $b_{r}\left(d_{r}\right)$ and $b_{r}^{\dagger}\left(d_{r}^{\dagger}\right)$ are creation and annihilation operators for fermions (antifermions), which satisfy the following relations:

$\left\{b_{s}(p), b_{r}^{\dagger}\left(p^{\prime}\right)\right\}=\left\{d_{s}(p), d_{r}^{\dagger}\left(p^{\prime}\right)\right\}=(2 \pi)^{3} \delta_{s r} \delta^{(3)}\left(\mathbf{p}-\mathbf{p}^{\prime}\right)$.

Now by using Lagrangian (15) and Fig. 1, the first order photon-neutrino Hamiltonian interaction is given by

$$
\begin{aligned}
H_{I}^{0}= & \int d \mathbf{q} d \mathbf{q}^{\prime} d \mathbf{p} d \mathbf{p}^{\prime}(2 \pi)^{3} \delta^{3}\left(\mathbf{q}^{\prime}+\mathbf{p}^{\prime}-\mathbf{p}-\mathbf{q}\right) \\
& \times \exp \left[i t\left(q^{\prime 0}+p^{0}-q^{0}-p^{0}\right)\right]\left(b_{r^{\prime}}^{\dagger} a_{s^{\prime}}^{\dagger}\left(\mathcal{M}_{1}+\mathcal{M}_{2}\right) a_{s} b_{r}\right),
\end{aligned}
$$

where

$$
\begin{aligned}
& \mathcal{M}_{1}+\mathcal{M}_{2}=-\frac{1}{8} e^{2} g_{w}^{2} \int \frac{d^{4} k}{(2 \pi)^{4}} D_{\alpha \beta}(q-k) \bar{U}_{r^{\prime}}\left(q^{\prime}\right) \gamma^{\alpha}\left(1-\gamma_{5}\right) \\
& \times S_{F}\left(k+p-p^{\prime}\right)\left[\xi_{s^{\prime}} S_{F}(k+p) \xi_{s}+\notin_{s} S_{F}\left(k-p^{\prime}\right) \notin_{s^{\prime}}\right] \\
& \times S_{F}(k) \gamma^{\beta}\left(1-\gamma_{5}\right) U_{r}(q)
\end{aligned}
$$

here $D_{\alpha \beta}$ and $S_{F}$ are boson and fermion propagators, $g_{W}$ is the weak coupling constant, and we choose our notation $d \mathbf{q}=d^{3} q /\left[(2 \pi)^{3} 2 q^{0}\right]$, and the same for $d \mathbf{p}, d \mathbf{p}^{\prime}$ and $d \mathbf{q}^{\prime}$. By using the above result, $H_{I}^{0}$, and Eq. (14), we are ready to find the commutator in the photon-neutrino forward scattering term,

$$
\begin{aligned}
{\left[H_{I}^{0}, D_{i j}^{0}(\mathbf{k})\right]=} & \int d \mathbf{q} d \mathbf{q}^{\prime} d \mathbf{p} d \mathbf{p}^{\prime}(2 \pi)^{3} \delta^{3}\left(\mathbf{q}^{\prime}+\mathbf{p}^{\prime}-\mathbf{p}-\mathbf{q}\right) \\
& \times\left(\mathcal{M}_{1}+\mathcal{M}_{2}\right)(2 \pi)^{3}\left[b_{r^{\prime}}^{\dagger} b_{r} a_{s^{\prime}}^{\dagger} a_{s} 2 p^{0} \delta_{i s} \delta^{3}\right. \\
& \left.\times(\mathbf{k}-\mathbf{p})-b_{r^{\prime}}^{\dagger} b_{r} a_{i}^{\dagger} a_{s} 2 p^{\prime 0} \delta_{j s^{\prime}} \delta^{3}\left(\mathbf{k}-\mathbf{p}^{\prime}\right)\right] .
\end{aligned}
$$

On using the above expectation values and the operator expectation values [1],

$$
\begin{aligned}
& \left\langle a_{1} a 2 \ldots b_{1} b_{2} \ldots\right\rangle=\left\langle a_{1} a_{2} \ldots\right\rangle\left\langle b_{1} b_{2} \ldots\right\rangle, \\
& \left\langle a_{s^{\prime}}^{\dagger}\left(p^{\prime}\right) a_{s}(p)\right\rangle=2 p^{0}(2 \pi)^{3} \delta^{3}\left(\mathbf{p}-\mathbf{p}^{\prime}\right) \rho_{s s^{\prime}}(\mathbf{x}, \mathbf{p}), \\
& \left\langle b_{r^{\prime}}^{\dagger}\left(q^{\prime}\right) b_{r}(q)\right\rangle=(2 \pi)^{3} \delta^{3}\left(\mathbf{q}-\mathbf{q}^{\prime}\right) \delta_{s s^{\prime}} \frac{1}{2} f_{v}(\mathbf{x}, \mathbf{q}),
\end{aligned}
$$

it follows that

$$
\begin{aligned}
& i\left\langle\left[H_{I}^{0}, D_{i j}^{0}(\mathbf{k})\right]\right\rangle=-\frac{i}{16} e^{2} g_{w}^{2} \int d \mathbf{q}\left(\rho_{s^{\prime} j}(\mathbf{k}) \delta_{i s}-\rho_{i s}(\mathbf{k}) \delta_{j s^{\prime}}\right) f_{v}(\mathbf{x}, \mathbf{q}) \\
& \quad \times \int \frac{d^{4} l}{(2 \pi)^{4}} D_{\alpha \beta}(q-l) \bar{U}_{r}\left(q^{\prime}\right) \gamma^{\alpha}\left(1-\gamma_{5}\right) S_{F}(l) \\
& \quad \times\left[\Varangle_{s^{\prime}}, S_{F}(l+k) \Varangle_{s}+\Varangle_{s} S_{F}(l-k) \phi_{s^{\prime}}\right] \\
& \quad \times S_{F}(l) \gamma^{\beta}\left(1-\gamma_{5}\right) U_{r}(q),
\end{aligned}
$$

where integrating on $l$ comes from the loop photon-neutrino interaction. Before we proceed let us give a short discussion of $f_{v}(\mathbf{x}, \mathbf{q})$; this quantity represents the number density of the neutrinos of momentum $\mathbf{q}$ per unit volume $(\mathrm{C} v \mathrm{~B}$ distribution function) so that

$n_{v}(\mathbf{x})=\frac{1}{(2 \pi)^{3}} \int d^{3} q f_{v}(\mathbf{x}, \mathbf{q})$,

where $n_{v}(\mathbf{x})$ is the local neutrino number density. Also the average bulk momentum of the neutrinos in the $\hat{i}$-direction is given by

$$
\left\langle q_{i}\right\rangle=\frac{1}{n_{\nu}(\mathbf{x})} \int \frac{d^{3} q}{(2 \pi)^{3}} f_{v}(\mathbf{x}, \mathbf{q}) q_{i}=\tilde{q}_{i}=m_{\nu} \tilde{v}_{i}, i=x, y, z,
$$

where $\tilde{\mathbf{q}} \neq 0$ is the average bulk momentum of neutrino and $|\tilde{\mathbf{v}}|$ is the neutrino bulk velocity which we consider $|\tilde{\mathbf{v}}|<\Delta T / T$. With the help of dimensional regularization and the Feynman parameters, we will go forward to obtain the leading order term of the right-hand side of the above equation, then

$$
\begin{aligned}
& i\left\langle\left[H_{I}^{0}, D_{i j}^{0}(\mathbf{k})\right]\right\rangle=-\frac{1}{16} \frac{1}{4 \pi^{2}} e^{2} g_{w}^{2} \\
& \quad \times \int d \mathbf{q}\left(\rho_{s^{\prime} j}(\mathbf{k}) \delta_{i s}-\rho_{i s}(\mathbf{k}) \delta_{j s^{\prime}}\right) f_{v}(x, q) \\
& \quad \times \int_{0}^{1} d y \int_{0}^{1-y} d z \frac{(1-y-z)}{z M_{W}^{2}} \bar{U}_{r}(q)\left(1+\gamma_{5}\right)\left(2 z q \epsilon_{s^{\prime}} \cdot \epsilon_{s}\right. \\
& \left.\quad+2 z\left(\notin_{s^{\prime}} \mathbf{q} \cdot \epsilon_{s}+\notin_{s} \mathbf{q} \cdot \epsilon_{s^{\prime}}\right)+(3 y-1) \not k\left(\notin_{s} \notin_{s^{\prime}}-\notin_{s^{\prime}} \notin_{s}\right)\right) U_{r}(q) .
\end{aligned}
$$

Here we use the gamma-matrix identity $A B=2 A \cdot B-B A$, the polarization vector properties $k . \epsilon_{i}=0$ and $\epsilon_{i} . \epsilon_{j}=-\delta_{i j}$. Now everything is ready to see the time evolution of the Stokes parameters as well as each polarization state of CMB photons. We are interested in $V$ parameter which gives the contribution of the circular polarization, by considering Eqs. (14) and (29), $d V / d t$ is given as follows:

$$
\begin{aligned}
& \frac{d V(\mathbf{x}, \mathbf{k})}{d t} \approx \frac{1}{6} \frac{1}{(4 \pi)^{2}} \frac{e^{2} g_{w}^{2}}{M_{W}^{2} k^{0}} \int d \mathbf{q} f_{v}(x, q) \bar{U}_{r}(q)\left(1+\gamma_{5}\right) \\
& \quad \times\left[\left(\notin_{1} q . \epsilon_{1}-\notin_{2} q . \epsilon_{2}\right) Q(\mathbf{k})-\left(\notin_{1} q . \epsilon_{2}+\notin_{2} q . \epsilon_{1}\right) U(\mathbf{k})\right] U_{r}(q),
\end{aligned}
$$

we should remind the reader that $U(k)$ is one of the Stokes parameters which represents linear polarization, while $U_{r}$ is Dirac spinor. We neglect the terms of $1 / M_{w}^{4}$ order and smaller. In order to proceed, let us introduce Dirac spinors 
and our frame work in more detail. $\gamma^{\mu}, \gamma^{5}$, and $U_{r}(q)$ are given by

$\gamma^{\mu}=\left(\begin{array}{cc}0 & \sigma^{\mu} \\ \bar{\sigma}^{\mu} & 0\end{array}\right), \quad \gamma^{5}=\left(\begin{array}{cc}-I & 0 \\ 0 & I\end{array}\right), \quad U_{r}(q)=\left(\begin{array}{c}\sqrt{p . \sigma} \xi^{r} \\ \sqrt{p . \bar{\sigma}} \xi^{r}\end{array}\right)$

where $\sigma^{\mu}=(1, \sigma), \bar{\sigma}^{\mu}=(1,-\sigma)$ and $\xi$ is two-component spinor normalized to unity. By assuming $E_{v}>>m_{v}$, the neutrino spinors are given by

$U_{r}(q)=\sqrt{2 E_{v}}\left(\begin{array}{l}\frac{1}{2}\left(1-\mathbf{p} . \sigma / E_{v}\right) \xi^{r} \\ \frac{1}{2}\left(1+\mathbf{p} . \sigma / E_{v}\right) \xi^{r}\end{array}\right)$.

Also the following equations are useful:

$$
\begin{aligned}
& \bar{U}_{r}(q) \gamma^{\mu} U_{s}(q)=2 q^{\mu} \delta^{r s}, \\
& \quad \times \frac{1}{2} \sum_{r} \bar{U}_{r}(q) \gamma^{\mu}\left(1-\gamma^{5}\right) U_{r}(q)=2 q^{\mu} .
\end{aligned}
$$

Then by making average on the spin of the neutrinos, $\frac{1}{2} \sum_{r}$, and substituting the above equations in Eq. (30), we arrive at

$$
\begin{aligned}
& \frac{d V(\mathbf{x}, \mathbf{k})}{d t} \approx \frac{\sqrt{2}}{3 \pi k^{0}} \alpha G^{F} \int d \mathbf{q} f_{v}(x, q) \\
& \times\left[\left(q . \epsilon_{1} q . \epsilon_{1}-q . \epsilon_{2} q . \epsilon_{2}\right) Q(\mathbf{k})\right. \\
& \left.-\left(q . \epsilon_{1} q . \epsilon_{2}+q . \epsilon_{2} q . \epsilon_{1}\right) U(\mathbf{k})\right],
\end{aligned}
$$

where

$$
G^{F}=\frac{\sqrt{2}}{8} \frac{g_{W}^{2}}{M_{W}^{2}} \approx 1.16 \times 10^{-5}(\mathrm{GeV})^{-2}, \quad \alpha=\frac{e^{2}}{4 \pi}=1 / 137 .
$$

This equation contains an integration on the neutrino momentum which we should determine. Next by using Eqs. (27), (28), and the calculation given in Appendix A, the time evolution of the $V$ mode is given by

$$
\begin{aligned}
& \frac{d V(\mathbf{x}, \mathbf{k})}{d t} \approx \frac{\sqrt{2}}{3 \pi} \frac{|\tilde{\mathbf{q}}|}{k^{0}} \alpha G^{F} n_{\nu}(\mathbf{x})|\tilde{\mathbf{v}}| \\
& \times\left[\left(\tilde{\hat{\mathbf{q}}} \cdot \hat{\epsilon}_{1} \tilde{\hat{\mathbf{q}}} \cdot \hat{\epsilon} \hat{\epsilon}_{1}-\tilde{\hat{\mathbf{q}}} \cdot \hat{\epsilon} 2 \hat{\mathbf{q}} \cdot \hat{\epsilon}_{2}\right) Q(\mathbf{k})-\left(\tilde{\hat{\mathbf{q}}} \cdot \hat{\epsilon} \hat{\epsilon}_{1} \tilde{\hat{\mathbf{q}}} \cdot \hat{\epsilon}_{2}+\tilde{\hat{\mathbf{q}}} \cdot \hat{\epsilon}_{2} \tilde{\hat{\mathbf{q}}} \cdot \hat{\epsilon}_{1}\right) U(\mathbf{k})\right] .
\end{aligned}
$$

We proceed by considering $k^{0} \approx T_{\gamma}$ and also avoid the angular distribution of each mode

$V(\mathbf{x}, k)=\int \frac{d \Omega}{4 \pi} V(\mathbf{x}, \mathbf{k}), \quad k=|\mathbf{k}|=k^{0}$,

where $d \Omega$ is the differential solid angle. We consider $Q(\mathbf{x}, k)$, $U(\mathbf{x}, k)$, and $I(\mathbf{x}, k)$ modes in the same way as well as $V(\mathbf{x}, k)$. Then

$\frac{d V}{d t}(\mathbf{x}, k) \approx \frac{\sqrt{2}}{3 \pi} \frac{|\tilde{\mathbf{q}}|}{k^{0}} \alpha G^{F} n_{v}(\mathbf{x})|\tilde{\mathbf{v}}|\left(C_{U}+C_{Q}\right)$,

where

$C_{U}=-\int \frac{d \Omega}{4 \pi}\left(\tilde{\hat{\mathbf{q}}} \cdot \hat{\epsilon}_{1} \tilde{\hat{\mathbf{q}}} \cdot \hat{\epsilon}_{2}+\tilde{\hat{\mathbf{q}}} \cdot \hat{\epsilon}_{2} \tilde{\hat{\mathbf{q}}} \cdot \hat{\epsilon}_{1}\right) U(\mathbf{x}, \mathbf{k})$,
$C_{Q}=\int \frac{d \Omega}{4 \pi}\left(\tilde{\hat{\mathbf{q}}} \cdot \hat{\hat{\epsilon}_{1}} \tilde{\hat{\mathbf{q}}} \cdot \hat{\epsilon}_{1}-\tilde{\hat{\mathbf{q}}} \cdot \hat{\epsilon}_{2} \tilde{\hat{\mathbf{q}}} \cdot \hat{\epsilon}_{2}\right) Q(\mathbf{x}, \mathbf{k})$

In general $|\tilde{\mathbf{q}}|$ depends on time as well as redshift and to obtain this dependence, the Boltzmann equation for the distribution function of the neutrino should be solved. But here we consider $|\tilde{\mathbf{q}}|(z)=(1+z)|\tilde{\mathbf{q}}|_{0}$ where $|\tilde{\mathbf{q}}|_{0}$ is the value of the average perturbation bulk momentum of the cosmic neutrino background at present. To estimate the $V$, we integrate over the comoving time $\int d t=\int d z / H(z)$, where the redshift $z \in\left[0,10^{3}\right]$, the Hubble function $H(z)=$ $\left.H_{0}\left[\Omega_{M}(z+1)^{3}+\Omega_{\Lambda}\right)\right]^{1 / 2}$ for $\Omega_{M} \simeq 0.3, \Omega_{\Lambda} \simeq 0.7$ and $H_{0}=75 \mathrm{~km} / \mathrm{s} / \mathrm{Mpc}$, and the temperature $T_{\gamma, v}=T_{0, \gamma, v}(1+z)$ $\left[T_{0, \gamma} \approx 2.725 \mathrm{~K}^{\circ}=2.349 \times 10^{-4} \mathrm{eV}=(0.511 \mathrm{~cm})^{-1}\right]$ in the standard cosmology [38]. Also $n_{v}=n_{v}^{0}(1+z)^{3}$ where $n_{v}^{0}(\mathbf{x}) \sim 50 / \mathrm{cm}^{3}$ is today's $\mathrm{C} v \mathrm{~B}$ number density. Finally $V$ is given by

$$
\begin{aligned}
V(\mathbf{x}, k) \approx & \frac{\sqrt{2}}{3 \pi} \frac{|\tilde{\mathbf{q}}|}{k^{0}} \alpha G^{F} n_{v}^{0}(\mathbf{x})|\tilde{\mathbf{v}}| \\
& \times \int_{0}^{1,000} \frac{(1+z)^{3} d z}{\left(\Omega_{M}(z+1)^{3}+\Omega_{\Lambda}\right)^{1 / 2}}\left(C_{U}+C_{Q}\right),
\end{aligned}
$$

where we can substitute

$$
\begin{aligned}
H_{0}^{-1} & \approx 6 \times 10^{41}(\mathrm{GeV})^{-1}, \quad T_{0, v} \approx 1.67 \times 10^{-13} \mathrm{GeV}, \\
T_{0, \gamma} & \approx 2.34 \times 10^{-13} \mathrm{GeV},
\end{aligned}
$$

into Eq. (41) and integrate on redshift $z$. By assuming the independence $\left(C_{U}+C_{Q}\right)$ from redshift, we arrive

$V(\mathbf{x}, k) \approx 2 \times 10^{2} \frac{\tilde{q}}{k} \frac{n_{\nu}^{0}(\mathbf{x})}{50 / \mathrm{cm}^{3}}|\tilde{\mathbf{v}}|\left(C_{U}+C_{Q}\right)$,

where $\tilde{q}=|\tilde{\mathbf{q}}|_{0}$ and $k \simeq T_{0, \gamma}$ are the values of the average bulk momentum of the neutrinos and average energy of $\mathrm{CMB}$ at present universe. Now we need to make some estimations of $C_{U}$ and $C_{Q}$ as well as the $U$ and $Q$ polarization modes. A first approximation for these quantities is given by $V / I<\delta T / T$ [39]; this implies that $\frac{\tilde{q}}{k}|\tilde{\mathbf{v}}|\left(C_{U}+C_{Q}\right) / I$ should be smaller than $10^{-7}$. Let us investigate $C_{U}$ and $C_{Q}$ more precisely. A Fourier transform over the spatial dependence $\mathbf{x}$ of Eq. (43) gives

$V(\mathbf{K}, k) \approx 2 \times 10^{2} \frac{\tilde{q}}{k} \frac{n_{v}^{0}(\mathbf{K})}{50 / \mathrm{cm}^{3}}\left(C_{U}(\mathbf{K}, k)+C_{Q}(\mathbf{K}, k)\right)$,

where $\mathbf{K}$ is the Fourier conjugate of $\mathbf{x}$. For scalar perturbations, the local bulk velocity of electrons and baryons $\mathbf{v}_{b}$ has the same direction as $\mathbf{K}\left(\mathbf{v}_{b} \propto \mathbf{K}\right)$ [1,40]. For the rest of paper by neglecting the effects of tensor perturbations, we also consider the local bulk velocity of the neutrino in the $\mathbf{K}$ 


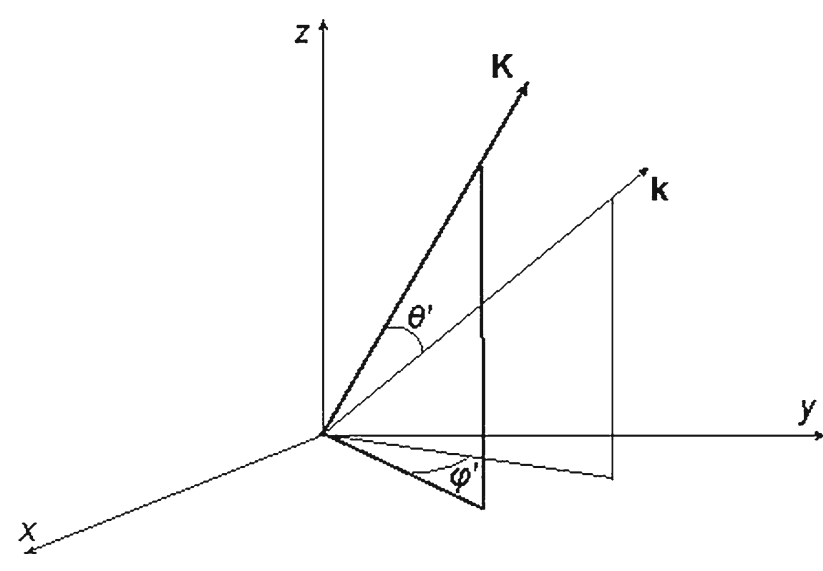

Fig. 2 Angles and directions to determine the angular dependence of Eq. (44)

direction $(\hat{\tilde{\mathbf{q}}} \propto \hat{\mathbf{K}})$. Assuming that $\theta^{\prime}$ is the angle between $\tilde{\mathbf{q}}$ or $\mathbf{K}$ and $\mathbf{k}$ [see Fig. 2], then we have

$C_{U}(\mathbf{K}, k)=-\int \frac{d \Omega^{\prime}}{4 \pi}\left(\sin ^{2} \theta^{\prime} \sin 2 \phi^{\prime}\right) U(\mathbf{K}, \mathbf{k})$,

$C_{Q}(\mathbf{K}, k)=\int \frac{d \Omega^{\prime}}{4 \pi}\left(\sin ^{2} \theta^{\prime} \cos 2 \phi^{\prime}\right) Q(\mathbf{K}, \mathbf{k})$,

where $k=|\mathbf{k}|$. Then one can expand the incident intensities $U$ and $Q$ in spherical harmonics (around $\mathbf{K}$ direction) as follows:

$$
\begin{aligned}
& U(\mathbf{K}, \mathbf{k})=\sum_{l m} \mathfrak{u}_{l m}(k) Y_{l, m}\left(\theta^{\prime}, \phi^{\prime}\right), \\
& Q(\mathbf{K}, \mathbf{k})=\sum_{l m} \mathfrak{q}_{l m}(k) Y_{l, m}\left(\theta^{\prime}, \phi^{\prime}\right) .
\end{aligned}
$$

By Eqs. (43)-(47), the photon-neutrino scattering generates circular polarization $\mathrm{CMB}$ from initially linear polarized $\mathrm{CMB}$ if this linear intensity $\left(C_{U}\right.$ and $\left.C_{Q}\right)$ at a given point as a function of direction has no-zero component $Y_{22}$.

$$
\begin{aligned}
C_{U}(\mathbf{K}, k) & =-\frac{2}{\pi}\left(\sqrt{\frac{2 \pi}{15}}\right) \mathfrak{u}_{22}(k), \\
C_{Q}(\mathbf{K}, k) & =\frac{2}{\pi}\left(\sqrt{\frac{2 \pi}{15}}\right) \mathfrak{q}_{22}(k) .
\end{aligned}
$$

As a result of this calculation, the exactly value of $V$ parameter in each $\mathbf{K}$ mode depends to the quadruple components of the incident intensity distribution and the square of the perturbation neutrino bulk velocity.

\section{The anisotropy of the photon distribution}

The previous section has been devoted to the right side of the Boltzman equation, and collision and scattering terms. In this section we discuss the left side term, which describes the propagation of photons in the background space-time. As discussed in $[1-4,38]$, the first order deviation from flat spacetime in the metric perturbation leads to an incommodiously for the photon $\mathrm{CMB}$ distribution function which is necessary to generate unpolarized CMB due to Thomson scattering. To proceed, we expand the photon distribution function $f_{\gamma}(\mathbf{k}, \mathbf{x})$ about its zero-order Bose-Einstein value as follows:

$$
\begin{aligned}
f_{\gamma}(\mathbf{k}, \mathbf{x}, t) & =\left[\exp \left\{\frac{k}{T_{\gamma}(t)(1+\Theta(\mathbf{k}, \mathbf{x}, t))}\right\}-1\right]^{-1} \\
& \simeq f_{\gamma}^{0}+k \frac{\partial f_{\gamma}^{0}}{\partial k} \Theta(\mathbf{k}, \mathbf{x}, t),
\end{aligned}
$$

where $f_{\gamma}^{0}=\left[\exp \left\{\frac{k}{T_{\gamma}}\right\}-1\right]^{-1}$ and $\Theta=\delta T / T$. Here the zeroorder temperature $T_{\gamma}(t)$ is a function of time only, not space. The perturbation to the distribution function is characterized by $\Theta(\mathbf{k}, \mathbf{x}, t)$. In the smooth zero-order universe, photons are distributed homogeneously, that is, $T_{\gamma}$ is independent of $\mathbf{x}$ and isotropic, so $T_{\gamma}$ is independent of the direction of propagation $\mathbf{k}$. We decomposed the perturbation $\Theta$ into a sum over Legendre polynomials,

$\Theta(\mathbf{x}, \mathbf{k}, t)=\sum_{l} \Theta_{l}(k) \mathcal{P}_{l}(\mu)$,

where $\mu$ is the dot product of the wave vector $\mathbf{k}$ and the direction of propagation [38] where in conjugate coordinates

$\Theta(\mathbf{K}, \mathbf{k}, t)=\sum_{l} \Theta_{l}(k) \mathcal{P}_{l}(\mathbf{K} . \mathbf{k})$.

By using Eqs. $(50,54)$, we can expand the intensity of the $\mathrm{CMB}$ radiation as

$$
\begin{aligned}
I(\mathbf{K}, \mathbf{k}, t) & \simeq I_{0}(k, t)+4 k \frac{\partial I_{0}}{\partial k} \Delta I(\mathbf{K}, \mathbf{k}, t)+\cdots \\
& \simeq=I_{0}(k, t)(1+4 \Theta(\mathbf{K}, \mathbf{k}, t)),
\end{aligned}
$$

where $\Delta I(\mathbf{K}, \mathbf{k}, t)$ depends on $\Theta(\mathbf{K}, \mathbf{k}, t)$, so this quantity can represent a sum over Legendre polynomials like Eq. (54),

$\Delta I(\mathbf{K}, \mathbf{k}, t)=\sum_{l} \Delta I_{l}(k) \mathcal{P}_{l}(\mathbf{K} . \mathbf{k})$.

On the other hand, at first glance, the Compton scattering is a perfect mechanism for producing polarized radiation. But to produce polarized radiation, the incoming radiation must have a nonzero quadruple component $[1,38]$,

$$
\begin{aligned}
& \frac{d}{d t} \Delta Q(\mathbf{K}, \mathbf{k}, t) \approx \sigma_{T} \bar{n}_{e} \Delta I_{2}(\mathbf{K}, k, t) \sin ^{2} \theta^{\prime} \cos 2 \phi^{\prime}, \\
& \frac{d}{d t} \Delta U(\mathbf{K}, \mathbf{k}, t) \approx-\sigma_{T} \bar{n}_{e} \Delta I_{2}(\mathbf{K}, k, t) \sin ^{2} \theta^{\prime} \sin 2 \phi^{\prime},
\end{aligned}
$$

where $\theta^{\prime}$ and $\phi^{\prime}$ are determined in Fig. 2 and

$$
\begin{aligned}
& \Delta Q(\mathbf{K}, \mathbf{k}, t)=\left(4 k \frac{\partial I_{0}}{\partial k}\right)^{-1} Q(\mathbf{K}, \mathbf{k}, t), \\
& \Delta U(\mathbf{K}, \mathbf{k}, t)=\left(4 k \frac{\partial I_{0}}{\partial k}\right)^{-1} U(\mathbf{K}, \mathbf{k}, t),
\end{aligned}
$$


here $\Delta Q(\Delta U)$ are dimensionless quantities which should be smaller than $\delta T / T, \bar{n}_{e}$ is the electron number density, and $I_{0}(k, t)$ is the unpolarized intensity of the CMB, which depends on $T_{\gamma}^{4}$. By substituting Eq. (55) into (41) and choosing the spherical coordinates for $\hat{\mathbf{k}}$ with the axis in the $\mathbf{K}$ direction (as shown in Fig. 2), we obtain the reasonable estimation for the $V$ mode in terms of $\Theta_{2}$ at present time, $t_{0}$ :

$$
\begin{aligned}
\Delta V & \left(\mathbf{K}, k, t_{0}\right) \\
\approx & 5 \times 10^{-6}|\tilde{\mathbf{v}}|\left(\frac{n_{v}^{0}(\mathbf{K})}{50 / \mathrm{cm}^{3}}\right)\left(\frac{\tilde{q}}{k}\right) \sigma_{T} H_{0}^{-1} \bar{n}_{e} \Delta I_{2}\left(\mathbf{K}, k, t_{0}\right) \\
& \times \int_{0}^{1,000} \frac{(1+z)^{3} d z}{\left(\Omega_{M}(z+1)^{3}+\Omega_{\Lambda}\right)^{1 / 2}} \\
& \times \int_{z}^{1,000} \frac{d z^{\prime}}{\left(\Omega_{M}\left(z^{\prime}+1\right)^{3}+\Omega_{\Lambda}\right)^{1 / 2}}
\end{aligned}
$$

where $\Delta V$ is defined as dimensionless quantity as well as $\Delta Q(\Delta U)$. Then by considering the relevant values for quantities which appear in the above equation and performing the integrations, we obtain

$$
\begin{aligned}
& \Delta V\left(\mathbf{K}, k, t_{0}\right) \\
& \quad \approx 5 \times 10^{2}|\tilde{\mathbf{v}}|^{2}\left(\frac{n_{v}^{0}(\mathbf{x})}{50 / \mathrm{cm}^{3}}\right)\left(\frac{m_{v}}{k}\right)\left(\frac{\bar{n}_{e}}{0.1 \mathrm{~cm}^{-3}}\right) \Delta I_{2}\left(\mathbf{K}, k, t_{0}\right) .
\end{aligned}
$$

The above equation is given for each mode of $\mathbf{K}$, but we are interested to measure the value of $V$-parameter in real space coordinates $\mathbf{x}$ (we have to take an inverse Furrier transform of the above equation) and then we have the two point function $\left\langle\Delta V\left(\mathbf{x}, k, t_{0}\right) \Delta V\left(\mathbf{x}, k, t_{0}\right)\right\rangle$ where

$\Delta V\left(\mathbf{x}, k, t_{0}\right)=\int \frac{d^{3} K}{(2 \pi)^{3}} e^{i \mathbf{K} \cdot \mathbf{x}} \Delta V\left(\mathbf{K}, k, t_{0}\right)$.

Before doing this transformation, we introduce $\xi(\mathbf{K})$, which is a random variable used to characterize the initial amplitude of each mode of $\mathbf{K}$. It has the following statistical property:

$$
\left\langle\xi^{*}\left(\mathbf{K}^{\prime}\right) \xi(\mathbf{K})\right\rangle=(2 \pi)^{3} \delta\left(\mathbf{K}^{\prime}-\mathbf{K}\right) p_{s}(K) .
$$

$p_{s}(K)$ is the so-called primordial scalar power spectrum (index " $s$ " shows scalar perturbations). As discussed in $[41,42]$, this quantity can be described as

$$
p_{s}(K)=p_{s}\left(K_{*}\right)\left(\frac{K}{K_{*}}\right)^{n_{s}-1},
$$

where $p_{s}\left(K_{*}\right)$ and $n_{*}$ are determined at pivot scale $K_{*} \simeq$ $0.05 / \mathrm{Mpc}$. In general $n_{s}$ and $p_{s}\left(K_{*}\right)$ depend on the pivot scale. As shown in the above equation, the simplest case, neglecting a possible tensor component, the initial conditions are characterized by only two parameters $n_{s}$ and $p_{s}\left(K_{*}\right)$. For simplicity, we consider scale-invariant (Harrison-Zel'dovich spectrum) case with $n_{s} \simeq 1$ and $p_{s}\left(K_{*}\right) \propto A_{s} \frac{2 \pi^{2}}{K_{*}^{3}}$. However, in principle, other initial condition are also possible. The total value of two point correlation function of the $\Delta V$ mode can be written as

$$
\begin{aligned}
C^{V}= & \left\langle\Delta V\left(\mathbf{x}, k, t_{0}\right) \Delta V\left(\mathbf{x}, k, t_{0}\right)\right\rangle \\
\simeq & \int \frac{d^{3} K}{(2 \pi)^{3}} p_{s}(K)\left|\Delta V\left(\mathbf{K}, k, t_{0}\right)\right|^{2} \\
\simeq & 2.5 \times 10^{11}|\tilde{\mathbf{v}}|^{4}\left(\frac{n_{v}^{0}(\mathbf{x})}{50 / \mathrm{cm}^{3}}\right)^{2} \\
& \times\left(\frac{m_{v} / 0.3 \mathrm{eV}}{k / T_{0, \gamma}}\right)^{2}\left(\frac{\bar{n}_{e}}{0.1 \mathrm{~cm}^{-3}}\right)^{2} C_{2}^{T},
\end{aligned}
$$

where

$C_{2}^{T}=\int \frac{d K}{4 \pi^{2}} K^{2} p_{s}(K)\left|\Delta I_{2}\left(\mathbf{K}, k, t_{0}\right)\right|^{2}$.

Here we obtain the two point correlation function of the $\Delta V$ mode as a function of the two point correlation function of the angular power spectrum of temperature fluctuations (for more details as regards $C_{l}^{T}$, see [1-3,41,42]). Finally from Eq. (62), the value of $C^{V}$ approximately is given by

$$
\begin{aligned}
C^{V} \simeq & 2.5 \times 10^{11}|\tilde{\mathbf{v}}|^{4}\left(\frac{n_{v}^{0}(\mathbf{x})}{50 / \mathrm{cm}^{3}}\right)^{2}\left(\frac{m_{v} / 0.3 \mathrm{eV}}{k / T_{0, \gamma}}\right)^{2} \\
& \times\left(\frac{\bar{n}_{e}}{0.1 \mathrm{~cm}^{-3}}\right)^{2} C_{2}^{T} .
\end{aligned}
$$

The above equation, $C^{v}$ depends on the value of perturbation momentum bulk of $\mathrm{C} \nu \mathrm{B}$ as $|\tilde{\mathbf{v}}|^{4}$. Here we consider $|\tilde{\mathbf{v}}| \leq$ $\delta T / T$ (see Appendix A) where the accepted value of $\delta T / T$ is about $10^{-5}$, so that we can estimate the maximum value of $C^{V}$ in terms of the two point correlation function of the angular power spectrum of temperature fluctuations $C_{2}^{T}$ as follows:

$$
\begin{aligned}
C^{V} \simeq & 2.5 \times 10^{-9}\left(\frac{|\tilde{\mathbf{v}}|}{\Delta T / T}\right)^{4}\left(\frac{n_{v}^{0}(\mathbf{x})}{50 / \mathrm{cm}^{3}}\right)^{2}\left(\frac{m_{v} / 0.3 \mathrm{eV}}{k / T_{0, \gamma}}\right)^{2} \\
& \times\left(\frac{\bar{n}_{e}}{0.1 \mathrm{~cm}^{-3}}\right)^{2}
\end{aligned}
$$

where the value of $C_{2}^{T}$ is about a $1,000 \mu \mathrm{K}$ square (see for example $[2,3,41,42])$. As a result, the maximum value of $C^{V}$ is about $2 \times 10^{-9}$ of the quadruple component of the temperature power spectrum $C_{2}^{T}$ or in the range of $n \mathrm{~K}$ square. Of course we can generalize the above calculation for the other components. In order to complete our calculation for $V$-parameter, we must consider the contribution of antineutrino-photon scattering too. The calculation shows that the contribution of antineutrino-photon scattering is the same as neutrino-photon scattering (with the same sign) by taking the number density of the antineutrino instead of the neutrino one; see Appendix B for more details. 


\section{After the last scattering}

By considering photon-neutrino scattering at the last scattering surface, we discuss and calculate the generation of the CMB's circular polarization. But we must remind the reader that, during the propagation from the last scattering surface to us, CMB photons encounter large-scale structures and undergo significant changes due to effects related to structure formation [43,44]. The polarization modifications may occur during propagating in this large structure formation. On the other hand the presence of thermal electrons and large-scale diffuse synchrotron emission toward galaxy clusters suggests the presence of the large-scale magnetic fields [45]. The presence of this large-scale magnetic field causes some modifications on the circular polarization of CMB due to the Compton scattering, which is discussed in [21-23]. The generation of circular polarization in the process of transferring of $\mathrm{CMB}$ within a large-scale magnetic field and structure, due to the presence of the electrons, is discussed in [20]. They show that the $V$ mode is about $10^{-9}$ for $\lambda=1 \mathrm{~cm}, z=1,000$, and for a length scale of about $1 \mathrm{Mpc}$ and a number density of electrons about 0.1 per $\mathrm{cm}^{3}$,

$$
V(k) \propto 10^{-9} \frac{\bar{n}_{e}}{0.1 \mathrm{~cm}^{-3}}\left(\frac{B}{10 \mu G}\right)^{2}\left(\frac{\lambda}{1 \mathrm{~cm}}\right)^{3} \frac{L}{1 \mathrm{Mpc}},
$$

smaller than the maximum value of the $V$ mode due to photon-neutrino scattering discussed in the previous section. As shown in Eq. (58), the value of the $V$ mode due to neutrino-photon scattering has a linear dependence on the wavelength $\lambda=1 / k^{0}$ unlike the cubic dependence of the result of [20].

Electron-photon scattering generates the linear polarization of $\mathrm{CMB}$ from unpolarized $\mathrm{CMB}$ [1] but this process does not give any contribution for the CMB's circular polarization in the absence of a magnetic field. The probability of the generation of circular polarization via Compton scattering in the presence of magnetic field is discussed in [23]. The maximum value of the $V$ mode is given by

$V(k) \propto 10^{5} \frac{\bar{n}_{e}}{0.1 \mathrm{~cm}^{-3}}\left(\frac{B}{10 \mu G}\right)\left(\frac{\lambda}{1 \mathrm{~cm}}\right)^{3} \frac{L}{1 \mathrm{Mpc}}\left(\frac{T_{e}}{m_{e}}\right)^{3}$,

where the maximum of $\frac{T_{e}}{m_{e}}$ is about $10^{-6}$. By considering $\frac{T_{e}}{m_{e}}$, the maximum value of the $V$ mode is about $10^{-13}$ where is smaller than result is given in Eq. (58). Also the above equation has the linear dependence on magnetic field (unlike the result of [20]) and the cubic dependence on the wavelength (like the result of [20]) while in the case of photon-neutrino scattering (58), the linear dependence on the wavelength appears. Of course we can determine the exact value for $V$ mode in Eqs. (58), (66) and (67) and we can be sure that the contribution of photon-neutrino scattering $V$ is the dominant contribution because there are unknown parameters in each equation such as the value of the large-scale magnetic field, $\delta T / T(\mathbf{x}, \mathbf{k})$ as well as $I(\mathbf{x}, \mathbf{k})$. But by considering relevant values for the large-scale magnetic field and anisotropies, we can find that the contribution of photon-neutrino scattering to generate CMB's circular polarization is dominant.

Another effect of the large-scale structure and magnetic field which needs to be discussed may appear as the circular polarization being converted to a linear one due to photonneutrino and Compton scattering, which means

$\frac{d Q(U)}{d t}(\mathbf{k}) \propto V(\mathbf{k})$.

First, we investigate the conversion of circular polarization to linear polarization via Compton scattering. The Compton scattering in the absence of a magnetic field does not give any term like (68); see section $I V$ in [1]. But the Compton scattering in the presence of a magnetic field gives [23]

$$
\begin{aligned}
Q(U)(\mathbf{k}) \propto & 10^{5} \frac{\bar{n}_{e}}{0.1 \mathrm{~cm}^{-3}}\left(\frac{B}{10 \mu G}\right)\left(\frac{\lambda}{1 \mathrm{~cm}}\right)^{3} \\
& \times \frac{L}{1 \mathrm{Mpc}}\left(\frac{T_{e}}{m_{e}}\right)^{3} V(\mathbf{k}) .
\end{aligned}
$$

If we substitute the value of the $V(\mathbf{k})$ from Eq. (41) and the relevant value of the parameters which appear in the above equation, the maximum value of $Q(U)(\mathbf{k})$ becomes very small. So we can neglect the conversion of the circular to linear polarization via Compton scattering. To investigate the conversion of the circular to linear polarization via photon-neutrino scattering, we use Eqs. (14) and (29), which give

$$
\begin{aligned}
\frac{d Q(\mathbf{k})}{d t} \approx & \frac{1}{6} \frac{1}{(4 \pi)^{2}} \frac{e^{2} g_{w}^{2}}{M_{W}^{2} k^{0}} \int d \mathbf{q} f_{v}(x, q)\left[\bar{U}_{r}(q)\left(1+\gamma_{5}\right)\right. \\
& \left.\times\left(\phi_{1} q . \epsilon_{1}-\phi_{2} q . \epsilon_{2}\right) U_{r}(q)\right] V(\mathbf{k})
\end{aligned}
$$

By substituting $V(\mathbf{k})$ from Eq. (41), the value of $Q(\mathbf{k})$ is proportional to $\left(G^{F}\right)^{2}$, which becomes very small. Thus, it is negligible.

\section{Conclusion}

In this letter, by approximately solving the first order of the quantum Boltzmann equation for the density matrix of a photon ensemble, and time evolution of the Stokes parameters, we show that the linear polarizations of the CMB can convert to circular polarizations by scattering the $\mathrm{CMB}$ photon on cosmic neutrinos background $\mathrm{C} \nu \mathrm{B}$. The maximum value of the $V$-Stokes parameter in $\mathbf{K}$ direction is given by (58) at frequencies of a few $\mathrm{GHz}$ with the linear dependence on the wavelength and the square dependence on the average bulk velocity of $\mathrm{C} v \mathrm{~B}$. To have a measurable quantity for cir- 
cular polarization, we calculate the $C^{V}=\langle V V\rangle$ angular power spectrum Eq. (65). By considering the value of average velocity bulk of $\mathrm{C} \nu \mathrm{N}$ about the fluctuation temperature $|\tilde{\mathbf{v}}| \leq \delta T / T$, the maximum value of $C^{V}$ is about $10^{-9}$ of the $C_{2}^{T}$ angular power spectrum or as well as in range of $\mathrm{nK}$ square. We should mention that we only try to estimate analytically the value of circular polarization due to $\mathrm{CMB}$ and $\mathrm{C} v \mathrm{~B}$ scattering. But to have the exactly value, we should numerically solve the Boltzmann equation for $\mathrm{CMB}$ and $\mathrm{C} \nu \mathrm{B}$ in during a model for expansion universe which leave it here.

It is expected that the polarization data, which will become available with the Planck 2014 data, provide valuable information on the nature of the CMB anomalies (with resolution in range of Nano-kelvin) [12] and there are also other high resolution polarization experiments such as ACTPol [46], PIXIE [47], SPIDER [48]. Of course we cannot exactly compare the effects of photon-neutrino scattering on the circular polarization of the CMB with other interactions without any knowledge about the pattern and distribution of the initial linear polarization but we can discuss about its maximum. Our value for circular polarization (58) is larger than the one which is given by [20] and comparable with the bound reported in [5]. In the work reported in [20], one shows that Faraday conversion process during the propagation of polarized CMB photons through regions of the large-scale structure containing magnetized relativistic plasma, such as galaxy clusters, will lead to a circularly polarized contribution of order $10^{-9}$ at frequencies of $10 \mathrm{GHz}$ with a cubic dependence on the wavelength (our result has a linear dependence on the wavelength) and a square dependence on the large-scale magnetic field. References [21,22] have argued that the presence of a large-scale magnetic field prior to equality can affect the photon-electron and the photon-ion scattering, this leads to the radiation becoming circularly polarized and the induced $V V$ angular power spectra have been computed. Their results are comparable with the result of [20]. In [23], the effect of the large scale of a magnetic field on the Compton scattering has been discussed, which leads to the generation of circular polarization for CMB. The band on the $V$ mode reported in [23] is very much smaller than our result and has a cubic dependence on the wavelength (our result has a linear dependence on the wavelength), a linear dependence on the large-scale magnetic field, and a cubic dependence on $T_{e} / m_{e}$. The band on the $V$ mode reported in [20] can be larger than our result if we have a large scale of magnetic field, of the order of $B>10 \mathrm{mG}$. Also in [23], one shows that CMB polarization acquires a small degree of circular polarization when the quantum electrodynamic sector of the standard model is extended by Lorentz non-invariant operators as well as non-commutativity. These results contain Lorentz non-invariant and non-commutativity parameters, and we do not know the exact values of them. In Ref. [24], it has been shown that circular polarizations of radiation fields can be generated from the effective Euler-Heisenberg Lagrangian of the order of $10^{-10} \mathrm{~K}$, which is very small. The transformation plane of the polarization into circular polarization via photon-photon interactions mediated by the neutral hydrogen background, $\gamma+\gamma+$ atom $\rightarrow \gamma+\gamma+$ atom, through completely forward processes, has been discussed in [25]. The ratio of circular to plane polarization intensities $V / Q$ is predicted to be at the level of several times $10^{-5}$ for some regions of angular size less than 1/300 and with large plane polarizations. So the value of the circular polarization (from $\mathrm{CMB}$ and $\mathrm{C} \nu \mathrm{B}$ forward scattering) seems to be large enough to detect. On the other hand, as we already mentioned, the detection of $\mathrm{C} \nu \mathrm{B}$ is hardly possible due to the weak interaction of the neutrinos with matter and due to their low energy; however, the measuring of CMB's circular polarization may give us a good experimental testifier for the cosmic neutrino back ground $\mathrm{C} \nu \mathrm{B}$.

Acknowledgments I would like to thank S. S. Xue and I. Motie for fruitful discussion.

Open Access This article is distributed under the terms of the Creative Commons Attribution License which permits any use, distribution, and reproduction in any medium, provided the original author(s) and the source are credited.

Funded by $\mathrm{SCOAP}^{3}$ / License Version CC BY 4.0.

\section{Appendix A: $\mathrm{C} v \mathrm{~B}$ distribution function and Integration in Eq. (34)}

The Boltzmann equation for the massive neutrino will be discussed in this section based on which is given in Ref. [38] chapter four. Here we also consider non-relativistic neutrino which is reasonable condition for neutrinos with mass larger than temperature. Since the recombination age $(T \sim 0.3 \mathrm{eV})$, the neutrinos with mass about $\sim 1 \mathrm{eV}$ are non-relativistic. It is perhaps simplest to derive the evolution equations for the massive neutrino by imposing conservation of the energymomentum tensor. Unlike the photons, the massive neutrino behaves like a fluid, so it can be described completely by the energy-momentum tensor. Nonetheless, here we will sacrifice simplicity and use the Boltzmann formalism to derive the neutrino equations. The conformal Newtonian gauge (also known as the longitudinal gauge) advocated by Mukhanov et al. [40] is a particularly simple gauge to use for the scalar mode of metric perturbations. The perturbations are characterized by two scalar potentials $\phi$ and $\psi$, which appear in the line element as

$d s^{2}=a^{2}(\tau)\left\{-(1+2 \psi) d \tau^{2}+(1+2 \phi) d x_{i} d x^{j}\right\}$.

It should be emphasized that the conformal Newtonian gauge is a restricted gauge since the metric is applicable only for the scalar mode of the metric perturbations; the vector and the 
tensor degrees of freedom are eliminated from the beginning. The time evolution of the neutrino distribution function $f_{v}$ is given (in the Boltzmann formalism) by

$\frac{\partial f_{v}}{\partial t}+\frac{\mathbf{p}_{i}}{a E_{v}} \frac{\partial f_{v}}{\partial x_{i}}-\frac{\partial f_{v}}{\partial E_{v}}\left(\frac{d a / d t}{a} \frac{p^{2}}{E_{v}}+\frac{p^{2}}{E_{v}} \frac{\partial \phi}{\partial t}+\frac{\mathbf{p}_{i}}{a} \frac{\partial \psi}{\partial x_{i}}\right)=0$.

Instead of assuming a form for $f_{v}$, we will take the zero moment equation by multiplying the above equation in $\int \frac{d^{3} p}{(2 \pi)^{3}}$, the first moment equation by multiplying the above equation in $\int \frac{d^{3} p}{(2 \pi)^{3}} \frac{\hat{\mathbf{p}}_{i} p}{E_{v}}$ and the second moment equation by multiplying the above equation by $\int \frac{d^{3} p}{(2 \pi)^{3}} \frac{\hat{\mathbf{p}}_{i} p}{E_{v}} \frac{\hat{\mathbf{p}}_{j} p}{E_{v}}$. Finally the following equations are obtained:

$$
\begin{aligned}
& \frac{\partial \delta_{v}}{\partial t}+\frac{1}{a} \frac{\partial v^{i}}{\partial x^{i}}+3 \frac{\partial \phi}{\partial t}=0, \\
& \frac{\partial v^{i}}{\partial t}+\frac{d a / d t}{a} v^{i}+\frac{1}{a} \frac{\partial \psi}{\partial x_{i}}=0, \\
& \frac{\partial v^{i} v^{j}}{\partial t}+2 \frac{d a / d t}{a} v^{i} v^{j}+\frac{1}{a}\left(\frac{\partial \psi}{\partial x_{i}} v^{j}+\frac{\partial \psi}{\partial x_{j}} v^{i}\right)=0,
\end{aligned}
$$

where $\delta_{v}=\left(n_{v}-n_{v}^{0}\right) / n_{v}^{0}, n_{v}^{0} \propto a^{-3}$ is the zero-order, homogeneous part of the density and $v^{i}$ is the neutrino bulk velocity in the $\hat{i}$-direction.

$v^{i}=\frac{1}{n_{v}} \int \frac{d^{3} p}{(2 \pi)^{3}} \frac{\hat{\mathbf{p}}_{i} p}{E_{v}} f_{v}$

$v^{i} v^{j}=\frac{1}{n_{v}} \int \frac{d^{3} p}{(2 \pi)^{3}} \frac{\hat{\mathbf{p}}^{i} p}{E_{v}} \frac{\hat{\mathbf{p}}^{j} p}{E_{v}} f_{\nu}$.

We should remind the reader that the second order terms in the perturbations in Eqs. (73) and (74) and the third order one in Eq. (75) are neglected. Let us finally rewrite Eqs. (73)-(75) in terms of the conformal time $\tau$ and the Fourier transforms (the conjugate quantities are shown like $\tilde{A}$ )

$\dot{\tilde{\delta}}_{v}+i K \tilde{v}+3 \dot{\tilde{\phi}}=0$

$\dot{\tilde{v}}+\frac{\dot{a}}{a} \tilde{v}+i K \tilde{\psi}=0$,

$\frac{\partial}{\partial \tau} \tilde{v}^{i} \tilde{v}^{j}+2 \frac{\dot{a}}{a} \tilde{v}^{i} \tilde{v}^{j}+i\left(K^{i} \tilde{v}^{j}+K^{j} \tilde{v}^{i}\right) \tilde{\psi}=0$,

where we have assumed that the velocity is irrotational so $v^{i}=\frac{K^{i}}{K} \tilde{v}$. Neglecting overall the neutrino chemical potential, the zero-order neutrino distribution function is given by the Fermi-Dirac distribution, $\frac{1}{e^{E_{v} / T_{v}}+1}$, where $E_{v}=$ $\left(|\mathbf{q}|^{2}+m_{v}^{2}\right)^{1 / 2}$. This distribution function describes $\mathrm{C} v \mathrm{~N}$ with zero bulk velocity, which is not our case. In our case the neutrinos are propagating with an average bulk velocity $\mathbf{v}(x)$ or as well as an average momentum $\tilde{\mathbf{q}}=m_{v} \mathbf{v}(x)$. As shown in the above equations $\mathbf{v}_{v}(x)$ should in order of the perturbations Eq. (78). Now let us return to the integration given by Eq. (34); we have an integration as below:

$$
\begin{aligned}
& \frac{1}{(2 \pi)^{3}} \int \frac{d^{3} q}{2 E_{v}} f_{v}\left[\left(\mathbf{q} \cdot \epsilon_{1} \mathbf{q} \cdot \epsilon_{1}-\mathbf{q} \cdot \epsilon_{2} \mathbf{q} \cdot \epsilon_{2}\right) Q(\mathbf{k})\right. \\
& \left.-\left(\mathbf{q} \cdot \epsilon_{1} \mathbf{q} \cdot \epsilon_{2}+\mathbf{q} \cdot \epsilon_{2} \mathbf{q} \cdot \epsilon_{1}\right) U(\mathbf{k})\right] .
\end{aligned}
$$

By using Eqs. (75) and (76), finally, we have

$$
\begin{aligned}
& \frac{1}{2} n_{v}(\mathbf{x})|\tilde{\mathbf{q}}||\tilde{\mathbf{v}}|\left[\left(\tilde{\hat{\mathbf{q}}} \cdot \epsilon_{1} \tilde{\hat{\mathbf{q}}} \cdot \epsilon_{1}-\tilde{\hat{\mathbf{q}}} \cdot \epsilon_{2} \tilde{\hat{\mathbf{q}}} \cdot \epsilon_{2}\right) Q(\mathbf{k})\right. \\
& \left.-\left(\tilde{\hat{\mathbf{q}}} \cdot \epsilon_{1} \tilde{\hat{\mathbf{q}}} \cdot \epsilon_{2}+\tilde{\hat{\mathbf{q}}} \cdot \epsilon_{2} \tilde{\hat{\mathbf{q}}} \cdot \epsilon_{1}\right) U(\mathbf{k})\right]
\end{aligned}
$$

Notice that, if the average neutrino bulk momentum vanishes, the above equation becomes zero. This means that the neutrino-photon forward scattering can generate circular polarization when the neutrino bulk momentum is not zero. Actually the neutrino bulk velocity depends on time and is not exactly in the $\mathbf{K}$ direction. But for simplicity we consider $v^{i}=\frac{K^{i}}{K} \tilde{v}$ and the average value of the neutrino bulk velocity is about perturbations $\tilde{v} \sim \frac{\Delta T}{T} \sim 10^{-5}$. We should remind the reader that the value given by Eq. (81) is not exactly the value of Eq. (34) but it can be maximum value of it.

\section{Appendix B: Antineutrino-photon scattering}

The first order photon-antineutrino Hamiltonian interaction is given

$$
\begin{aligned}
& H_{I}^{0}=\int d \mathbf{q} d \mathbf{q}^{\prime} d \mathbf{p} d \mathbf{p}^{\prime}(2 \pi)^{3} \delta^{3}\left(\mathbf{q}^{\prime}+\mathbf{p}^{\prime}-\mathbf{p}-\mathbf{q}\right) \\
& \quad \times \exp \left[i t\left(q^{\prime 0}+p^{\prime 0}-q^{0}-p^{0}\right)\right]\left(d_{r} a_{s^{\prime}}^{\dagger}\left(\mathcal{M}_{1}^{\prime}+\mathcal{M}_{2}^{\prime}\right) a_{s} d_{r^{\prime}}^{\dagger}\right),
\end{aligned}
$$

where the amplitude of the diagram shown in Fig. 3 and its crossing is given by

$$
\begin{aligned}
\mathcal{M}_{1}^{\prime}+\mathcal{M}_{2}^{\prime}= & \frac{1}{8} e^{2} g_{w}^{2} \int \frac{d^{4} l}{(2 \pi)^{4}} D_{\alpha \beta}(l-q) \overline{\mathcal{V}}_{r}(q) \gamma^{\alpha}\left(1-\gamma_{5}\right) \\
& \times S_{F}\left(p^{\prime}-p-l\right) \\
& \times\left[\notin_{s} S_{F}(-l-p) \notin_{s^{\prime}}+\notin_{s^{\prime}} S_{F}\left(p^{\prime}-l\right) \notin_{s^{\prime}}\right] \\
& \times S_{F}(-l) \gamma^{\beta}\left(1-\gamma_{5}\right) \mathcal{V}_{r^{\prime}}\left(q^{\prime}\right) .
\end{aligned}
$$

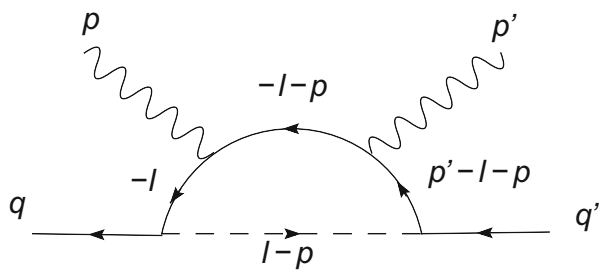

Fig. 3 The typical diagram of photon-antineutrino scattering 
Next we substitute the above equation into (22), then we take the average of this exsection value of antineutrino-photon forward scattering (similar to Eq. (26) for photon-neutrino scattering) and then we have

$$
\begin{aligned}
& i\left\langle\left[H_{I}^{0}, D_{i j}^{0}(\mathbf{k})\right]\right\rangle=\frac{i}{16} e^{2} g_{w}^{2} \int d \mathbf{q}\left(\rho_{s^{\prime} j}(\mathbf{k}) \delta_{i s}-\rho_{i s}(\mathbf{k}) \delta_{j s^{\prime}}\right) n_{\bar{v}}(x, q) \\
& \quad \times \int \frac{d^{4} l}{(2 \pi)^{4}} D_{\alpha \beta}(-q+l) \overline{\mathcal{V}}_{r}(q) \gamma^{\alpha}\left(1-\gamma_{5}\right) S_{F}(-l) \\
& \quad \times\left[\notin_{s} S_{F}(-l-k) \notin_{s^{\prime}}+\notin_{s^{\prime}} S_{F}(-l+k) \notin_{s}\right] S_{F}(-l) \gamma^{\beta}\left(1-\gamma_{5}\right) \mathcal{V}_{r}(q) .
\end{aligned}
$$

With the help of dimensional regularization and the Feynman parameters, we will proceed to obtain the leading order term of the right side of the above equation, then

$$
\begin{aligned}
i\langle[ & \left.\left.H_{I}^{0}, D_{i j}^{0}(\mathbf{k})\right]\right\rangle \\
= & -\frac{1}{16} \frac{1}{4 \pi^{2}} e^{2} g_{w}^{2} \int d \mathbf{q}\left(\rho_{s^{\prime} j}(\mathbf{k}) \delta_{i s}-\rho_{i s}(\mathbf{k}) \delta_{j s^{\prime}}\right) n_{\bar{v}}(x, q) \\
& \times \int_{0}^{1} d y \int_{0}^{1-y} d z \frac{(1-y-z)}{z M_{W}^{2}} \overline{\mathcal{V}}_{r}(q)\left(1+\gamma_{5}\right)\left(2 z \phi \epsilon_{s^{\prime}} \cdot \epsilon_{s}\right. \\
& \left.+2 z\left(\notin_{s^{\prime}} \mathbf{q} \cdot \epsilon_{s}+\notin_{s} \mathbf{q} \cdot \epsilon_{s^{\prime}}\right)-(3 y-1) \not k\left(\notin_{s} \xi_{s^{\prime}}-\notin_{s^{\prime}} \notin_{s}\right)\right) \mathcal{V}_{r}(q) .
\end{aligned}
$$

By use of the gamma-matrix identity, the polarization vector properties are $k . \epsilon_{i}=0$ and $\epsilon_{i} . \epsilon_{j}=-\delta_{i j}$. Finally $d V / d t$ is given as follows:

$$
\begin{aligned}
& \frac{d V(\mathbf{x}, \mathbf{k})}{d t} \approx+\frac{1}{6} \frac{1}{(4 \pi)^{2}} \frac{e^{2} g_{w}^{2}}{M_{W}^{2} k^{0}} \int d \mathbf{q} n_{\bar{\nu}}(x, q) \overline{\mathcal{V}}_{r}(q)\left(1+\gamma_{5}\right) \\
& \quad \times\left[\left(\notin_{1} q . \epsilon_{1}-\notin_{2} q . \epsilon_{2}\right) Q(\mathbf{x}, \mathbf{k})-\left(\notin_{1} q . \epsilon_{2}+\notin_{2} q . \epsilon_{1}\right) U(\mathbf{x}, \mathbf{k})\right] \mathcal{V}_{r}(q) .
\end{aligned}
$$

Also the equations below are useful:

$$
\begin{aligned}
& \overline{\mathcal{V}}_{r}(q) \gamma^{\mu} \mathcal{V}_{s}(q)=2 q^{\mu} \delta^{r s}, \\
& \quad \frac{1}{2} \sum_{r} \overline{\mathcal{V}}_{r}(q) \gamma^{\mu}\left(1 \pm \gamma^{5}\right) \mathcal{V}_{r}(q)=2 q^{\mu}
\end{aligned}
$$

If we substitute the above equation into (86), we will arrive at

$$
\begin{aligned}
\frac{d V(\mathbf{x}, \mathbf{k})}{d t} \approx & \frac{\sqrt{2}}{3 \pi} \frac{|\tilde{\mathbf{q}}|}{k^{0}} \alpha G^{F} n_{\bar{\nu}}(\mathbf{x}) \\
& \times\left[\left(\hat{\tilde{\mathbf{q}}} \cdot \hat{\epsilon}_{1} \hat{\tilde{\mathbf{q}}} \cdot \hat{\epsilon}_{1}-\hat{\tilde{\mathbf{q}}} \cdot \hat{\epsilon}_{2} \hat{\tilde{\mathbf{q}}} \cdot \hat{\epsilon}_{2}\right) Q(\mathbf{x}, \mathbf{k})\right. \\
& \left.-\left(\hat{\tilde{\mathbf{q}}} \cdot \hat{\epsilon}_{1} \hat{\tilde{\mathbf{q}}} \cdot \hat{\epsilon}_{2}+\hat{\tilde{\mathbf{q}}} \cdot \hat{\epsilon}_{2} \hat{\tilde{\mathbf{q}}} \cdot \hat{\epsilon}_{1}\right) U(\mathbf{x}, \mathbf{k})\right] .
\end{aligned}
$$

This equation is the same as (36), which means that antineutrino-photon scattering affects the circular polarization of CMB in the same way as the neutrino-photon scattering with the same value and sign.

\section{References}

1. A. Kosowsky, Annals Phys. 246, 49-85 (1996). arXiv:astro-ph/9501045

2. M. Zaldarriaga, U. Seljak, An all-sky analysis of polarization in the microwave background. Phys. Rev. D 55, 1830 (1997). arXiv:astro-ph/9609170

3. M. Zaldarriaga, D.N. Spergel, U. Seljak, Astrophys. J. 488, 1 (1997). arXiv:astro-ph/9702157

4. W. Hu, M.J. White, A CMB polarization primer. New Astron. 2, 323 (1997). arXiv:astro-ph/9706147

5. R.B. Partridge, J. Nowakowski, H.M. Martin, Nature 331, 146 (1988)

6. G.F. Smooth et al., Astrophys. J. Lett. 396(1), L1 (1992)

7. C.L. Bennett et al., Astrophys. J. Lett. 464, L1 (1996)

8. A.D. Miller et al., Astrophys. J. 521(2), L79 (1999)

9. A. Melchiorri et al., Astrophys. J. 536(2), L63 (2000)

10. S. Hanany et al., Astrophys. J. Lett. 545(1), L5 (2000)

11. G. Hinshaw et al., Astrophys. J. (Suppl Ser) 170(2), 288 (2007)

12. Planck Collaboration I, Planck 2013 results: Overview of Planck Products and Scientific Results (p01, p08) (2013). planck2013-p01, p08

13. N. Kaiser, MNRAS 202, 1169 (1983)

14. R. Crittenden, R. Davis, P. Steinhardt, Ap. J. 417, L13 (1993)

15. R.A. Frewin, A.G. Polnarev, P. Coles, Mon. Not. R. Ast. Soc. 266 , L21 (1994)

16. D. Harari, M. Zaldarriaga. Phys. Lett. B 319, 96 (1993)

17. J.D. Jackson, Classical Electrodynamic (Wiley, New York, 1998)

18. T.W. Jones, S.L. ODell, Astrophys J. 214, 522 (1977)

19. M. Ruszkowski, M.C. Begelman (2001) arXiv:astro-ph/0112090

20. A. Cooray, A. Melchiorri, J. Silk, Phys. Lett. B 554, 1 (2003). arXiv:astro-ph/0205214

21. M. Giovannini (2002). arXiv:hep-ph/0208152

22. M. Giovannini, K.E. Kunze, Phys. Rev. D 78, 023010 (2008). arXiv:astro-ph/0804.3380

23. M. Zarei, E. Bavarsad, M. Haghighat, R. Mohammadi, I. Motie, Z. Rezaei, Phys. Rev. D 81, 084035 (2010). arXiv:hep-th/0912.2993v5

24. I. Motie, S.-S. Xue, Europhys. Lett. 100, 17006 (2012). arXiv:hep-ph/1104.3555

25. R.F. Sawyer. arXiv:astro-ph.CO/1205.4969

26. C. L. Bennett, (2012), [arXiv:astro-ph/1212.5225].

27. Planck collaboration, 2013 submitted to Astronomy and Astrophysics (March 21, 2013)

28. A. Faessler, R. Hodak, S. Kovalenko, F. Simkovic, J. Phys. G 38, 075202 (2011). arXiv:hep-ph/1102.1799

29. R. Lazauskas, P. Vogel, C. Volpe, J. Phys. G 35, 1 (2008)

30. A. Ringwald, Y. Wong, J. Cosmol. Astroparticle Phys. 0412, 005 (2004)

31. R. Wigmans, Astropart. Phys. 19, 379 (2003)

32. T. Weiler, Phys. Rev. L 49, 234 (1982)

33. H.J. de Vega, O. Moreno, E. Moya de Guerra, M. Ramon Medrano, N.G. Sanchez. Nucl. Phys. B 866, 177 (2012). arXiv:hep-ph/1109.3452

34. F. Munyaneza, P.L. Biermann, Astron. Astrophys. 458, L9 (2006)

35. T.J. Weiler, Phys. Rev. D 63, 113015 (2001)

36. S. Betts et al. (2013). arXiv:astro-ph.IM/1307.4738v2

37. C. Itzykson, J.B. Zuber, Quantum Field Theory (McGraw-Hill, USA, 1980)

38. S. Dodelson, Modern Cosmology (Academic Press, London, 2003)

39. E.L. Wright (2003). arXiv:astro-ph/0305591

40. V.F. Mukhanov, H.A. Feldman, R.H. Brandenberger, Phys. Rep. 215, 203 (1992)

41. M. Tegmark et al., SDSS Collaboration. Phys. Rev. D 69, 103501 (2004). arXiv:astro-ph/0310723 
42. A. Cooray, Mod. Phys. Lett. A 20, 2503 (2005). arXiv:astro-ph/0503118

43. A. Cooray, in American Institute of Physics Conference Proceedings. arXiv:astro-ph/0203048

44. N. Aghanim, P.G. Castro, A. Melchiorri, J. Silk, Astron. Astrophys. 393, 381 (2002). arXiv:astro-ph/0203112

45. T.E. Clarke, P.P. Krongerg, B. Böhringer, Astrophys. J. Lett. L. 547, $111(2001)$
46. M.D. Niemack, P.A.R. Ade, J. Aguirre et al., in Proceedings of the SPIE, vol. 7741 (2010). arXiv:astro-ph.IM/1006.5049

47. A. Kogut et al. (2011). arXiv:astro-ph.CO/1105.2044

48. J.P. Filippini, P.A.R. Ade et al. arXiv:astro-ph.CO/1106.2158
(2011). 